\title{
Optimal Network Design for Consensus Formation: Wisdom of Networked Agents
}

\author{
Eugene S. Kitamura \\ Department of Computer Science, \\ National Defense Academy \\ Yokosuka, Japan
}

\author{
Akira Namatame \\ Department of Computer Science, \\ National Defense Academy \\ Yokosuka, Japan
}

\begin{abstract}
The wisdom of crowds refers to the phenomenon in which the collective knowledge of a community is greater than the knowledge of any individual. This paper proposes a network design for the fastest and slowest consensus formation under average node degree restrictions, which is one aspect of the wisdom of crowds concept. Consensus and synchronization problems are closely related to variety of issues such as collective behavior in nature, the interaction among agents as a matter of the robot control, and building efficient wireless sensor networks. However, designing networks with desirable properties is complex and it may pose a multi-constraint and multi-criterion optimization problem. For the purpose of realizing such efficient network topology, this paper presents an optimization approach to design networks for better consensus formation by focusing on the eigenvalue spectral of Laplacian matrix. In both the fastest and slowest networks presented, consensus is formed among local structures first, then on a global scale. This suggests that both local and global topology influence the networks dynamics. These findings are useful for those who seek to manage efficient consensus and synchronization in a setting that can be modeled as a multi-agent system.
\end{abstract} matrix

Keywords—wisdom of crowds; consensus problem; Laplacian

\section{INTRODUCTION}

There is a strong interest in many fields to answer the following questions. How do interacting individuals with micro-motives produce the desirable or undesirable outcomes at the aggregate level? How are interactions among agents that produce some regularities of interest at the macroscopic level identified? Most of our social activities are substantially free of centralized management, and although people may care about how it all comes out in the aggregate, people's own behaviors are typically motivated by self-interest. Therefore, in examining collective outcome, the observer shall draw heavily on the individual behaviors. It might be argued that understanding how individuals behave is sufficient to understand most parts of the social system. Although individual behaviors are important to understand, they are not sufficient to describe how a collection of agents generate desirable macroscopic outcome. To make the connection between microscopic behavior and macroscopic outcome of interests, the observer usually has to look at the system of interactions among agents described as the interaction network topology.

There are social systems for which it is difficult to understand how they work or to find better ways to make them work. For instance, social systems often produce inefficient outcomes at the aggregate level in a way that the individuals who comprise the system cannot evaluate or are not even aware of. When the system results in some undesirable outcome, the cause is often thought of as the members who comprise the system. The resulting outcome is observed as corresponding to the intentions of the members who compromise the system. It is not easy to tell from emergent phenomena just what the motives are behind individuals and how strong they are.

Social systems often result in the features of emergent properties, which are properties of the system in which separated components by themselves do not have. Other social phenomena are also viewed as emergence that have arisen from billions of small-scale and short-term decisions of interacting agents. Billions of people make billions of decisions everyday about many things. It often appears that the aggregation of these unmanaged individual decisions leads to unpredictable outcomes. Unintended consequences and side effects are closely related to emergent properties. In other words, the global or macroscopic functionality of a system is the sum of all side effects of all emergent properties.

People constantly interact with each other in different ways and for different purposes. Somehow these individual interactions produce some coherence at the aggregate level, and therefore, aggregation may generate structure and regularity. The individuals involved may have a very limited view of some part of the whole system but their activities are coordinated extensively and produce a desirable outcome at the aggregate level. These emergent properties are the result of not only the behavior of individuals but the interactions between them as well.

In his book, titled The Wisdom of Crowds, Surowiecki explores an idea that has profound implications: a large collection of people are smarter than an elite few, no matter how they are brilliant and better at solving problems, fostering innovation, coming to wise decisions, even predicting the future [1].

He explains the wisdom of crowds emerges only under the right conditions: (1) diversity, (2) independence, (3) decentralization, and (4) aggregation. His counterintuitive notion, rather than the madness of crowd such as herding or cascade as traditionally understood, suggests new insights for the issue on how complex social and economic activities should be organized. 
In contrast, Lorenza et al. [2] demonstrates by experimental evidence that even mild social influence can undermine the wisdom of crowd effect in simple estimation tasks. In the experiment, subjects could reconsider their response to factual questions after having received average or full information of the responses of other subjects. They compare subjects' convergence of estimates and improvements in accuracy over five consecutive estimation periods with a control condition in which no information about others' responses was provided. Although groups are initially wise, knowledge about the estimates of others narrows the diversity of opinions to such an extent that it undermines the wisdom of crowd effect. Especially the social influence effect diminishes the diversity of the crowd without improvements of its collective error.

This observation derives requirements for a more general model of network effects. Therefore a new area of research has emerged aiming at explaining the phenomena of strong positive or negative network effects in markets and their implications on market coordination and efficiency. However, the assumptions and simplifications implicitly used for modeling social interaction processes fail to explain the individual cognitive decision-making process as well as the network structure.

Consensus problems have a long history in computer science and control theory [3]. In networks of agents, consensus means to reach an agreement regarding a certain quantity of interest that depends on the state of all agents. A consensus algorithm is an interaction rule that specifies the information exchange between an agent and all of its neighbors on the network. The theoretical framework for solving consensus problems for networked systems was introduced by Olfati-Saber and colleagues [4].

Many of the essential features displayed by complex systems emerge from their underlying network structure. Whether optimization plays a key role in shaping the evolution of optimal networks is an important question [5]. One of the broadest areas of research, the optimization has a long history. Optimization is most often connected to a function that the system performs. In numerous cases the function is multifaceted. How network structure influences the global performance of such systems is probably the question that is posed most frequently in network research [6][7].

Here the following question is addressed: what topology fosters or dampens consensus or synchronization on networks? In this paper, such optimal topologies are constructed for any fixed number of nodes and links, by employing an evolutionary optimization procedure. The issue of the wisdom of networked agents in terms of convergence speed by formulating consensus problems is addressed. An evolutionary algorithm is used involving optimizing the eigenvalue ratio of the Laplacian matrix under the constraint of the average degree. Traditionally, optimization has a strict mathematical definition, which refers to obtaining the solutions that strictly optimize a well-defined objective function. Here a looser definition of the word is adopted by extending it to include a tendency of the system to improve its behavior as a result of a selection pressure based on artificially imposed fitness function. It comprises the variation principles or the survival-of-the-fittest principles that pervade biology and engineering, the foundational hypotheses of numerous computer algorithms, and the frameworks for addressing the improvement of efficiency in various contexts. Optimization in complex networks has a broad significance, incorporating static and dynamical properties and serving as an instrument to analyze and control the evolution and function of both natural and engineered systems.

In this paper, the fast consensus topology is introduced that is not Ramanujan for average degree less than 2 that has not been previously reported, generated with the genetic algorithm. Additionally, the fastest consensus is contrasted with the slow consensus topology, based on a heuristic model which was originally inspired by the evolutionary algorithm. Finally, a consensus of two clique topology connected by a single link will be examined. All of these networks display a topological priority in the progression of consensus, where consensus is formed initially with locally oriented agents that form close proximity. Formation of such topology by an agent society may be referred to as their wisdom, resulting in varying aggregate performances from a global perspective.

\section{NETWORK DESIGN FOR BETTER CONSENSUS}

It is broadly recognized that most complex systems in nature are organized as intricate network patterns. This observation has triggered an intense research effort aimed at understanding the organizing principles of these networks, their structural properties, and the interplay between topology and dynamics. Understanding the network structure of individual systems has led to tremendous advances in the past decade [8][9].

Most of the complex systems seen in real life also have associated dynamics, and the structural properties of such networks have to be linked with their dynamical behavior. While most of the initial effort was put into understanding the topological properties of networks, the interest has gradually shifted towards the analysis of the interplay between a topology and the dynamics of network components. In general, each element (node) in a network undergoes a dynamical process while coupled to other nodes. The system's collective behavior depends strongly on the efficiency of communication paths, which is in turn dictated by the underlying network topology. In this way, the network structure determines to a large extent the possibility of a coherent response.

Thus, the topology of the network remains static while the states of the nodes change dynamically. In this respect, one of the questions of obvious significance is whether there is a relation between the stability of the dynamics against small perturbations in the dynamical variables and the specific arrangement of the network's connections. If the perturbation decays quickly, so that it is unable to spread to the rest of the network, the network is said to be stable. Such a property is necessary if networks are to survive the noisy environment that characterizes the real world.

It has sometimes been argued that, networks with larger number of nodes, links and stronger interconnections are more stable. Such assertions are partly based on empirical observations, e.g., in ecology, where it has been found that more diverse and strongly connected ecosystems are more 
robust than their smaller, weakly connected counterparts. Some important processes studied within this framework include synchronization of the individual dynamical systems and consensus processes such as opinion formation. Studies like these have clarified that certain topological properties have strong impacts on the dynamics of the networks. In recent studies, the reason for the occurrence of synchronized networks became clear and the underlying network topology is important, however, little is known about what the best network topology is for synchronization [10][11].

Two principal approaches have contributed to understanding network structures so far. The first is an assembly mechanism that derives the structure of large complex networks from processes that describe the piecewise addition of nodes and links according to stochastic rules over time. Preferential attachment is an important mechanism in this category. The second approach is via optimization, thus assuming that a network structure observed in the real world represents the end point of some guided reorganization mechanism that aims at optimizing system performance during its evolution [12].

When modeling consensus, the underlying network restricts communication among agents where agents can exchange information only with the connected agents. There are many studies to analyze the influence of the network topology on the convergence speed of the iterative consensus algorithm.

Based on the concept of "The wisdom of crowds", Golub et al. [13] developed a social network approach to model consensus phenomenon. Their study basically uses an important model of network influence largely due to DeGroot who studied consensus problems in groups of experts who originated in statistics [14]. In their paper, Golub et al. examined one aspect of this broad theme: for which social network structures will a society of agents who communicate and update naïvely come to aggregate decentralized information completely and correctly. They focus on situations where there is some true state of nature that agents are trying to learn and each agent's initial belief is equal to the true state of nature plus some idiosyncratic zero-mean noise. The network structure of agents is described using a weighted network. Agents have beliefs about some common question of interestfor instance, the probability of some event. At each time step, agents communicate with their neighbors in the social network and update their beliefs. The updating process is simple. An agent's new belief is the average of his or her neighbors' beliefs from the previous period.

An outside observer who could aggregate all of the decentralized initial beliefs could develop an estimate of the true state that would be arbitrarily accurate in a large enough society. Golub et al. studied learning in a setting where agents receive independent noisy signals about the true value of a variable and then communicate it in a network. The agents naïvely update beliefs by repeatedly taking weighted averages of neighbors' opinions. They show that all opinions in a large society converge to the truth if and only if the influence of the most influential agent vanishes as the society grows. They also identify obstructions to this, including prominent groups, and provide structural conditions on the network ensuring efficient learning. Whether agents converge to the truth is unrelated to how quickly consensus is approached.

The consensus problem is also related to synchronization. Synchronization is the most prominent example of coherent behavior, and is a key phenomenon in systems of coupled oscillators as those characterizing most biological networks or physiological functions. Synchronous behavior is also affected by the network structure. The continuous range of stability of a synchronized state is a measure of the system's ability to yield a coherent response and to distribute information efficiently among its elements, while a loss of stability fosters pattern formation.

In recent studies, the reason for the occurrence of synchronized networks became clear and the underlying network topology turned out to be important. However, synchronization often occurs unexpectedly and little is known what the best network topology is for synchronization.

\section{OPTIMAL NETWORK DESIGN FOR BETTER CONSENSUS}

The analysis of consensus problems relies heavily on matrix theory and spectral graph theory [15]. The interaction topology of a network of agents is represented using an undirected graph $G$ with the set of nodes and edges. Neighbors of agent $i$ is denoted as $n_{i}$.

Consider a network of agents with the following dynamics:

$$
\dot{x}_{i}=\sum_{j \in N_{i}} \alpha_{i j}\left(x_{j}(t)-x_{i}(t)\right)
$$

where $a_{i j}$ is the weight of agent $i$ on agent $j$.

Here, reaching a consensus means asymptotically converging to the same internal state by way of an agreement characterized by the following equation:

$$
x_{1}=x_{2}=\cdots=x_{n}=\alpha
$$

Assuming that the underlying graph $G$ is undirected $\left(a_{i j}=a_{j i}\right.$ for all $i, j$ ), the collective dynamics converge to the average of the initial states of all agents:

$$
\alpha=\frac{1}{n} \sum_{i=1}^{n} x_{i}(0)
$$

The dynamics of system in (1) can be expressed as

$$
\dot{x}=-\mathbf{L} x(t) \text {. }
$$

$\mathbf{L}$ is the graph Laplacian matrix of the network $G$; the Laplacian matrix is defined as

$$
\mathbf{L}=\mathbf{D}-\mathbf{A},
$$

where $\mathbf{D}=\operatorname{diag}\left(d, d_{2}, \cdots, d_{n}\right)$ is the diagonal matrix with elements $d_{i}=\sum_{j} a_{i j}$ and $\mathbf{A}$ is the binary adjacency matrix ( $n$ $\times n$ matrix) with elements $a_{i j}$ for all $i, j$ where $a_{i j}$ is 1 if agent $i$ and agent $j$ is connected or 0 if they are disconnected. 
Notice that because the networks in this paper are undirected, $\mathbf{L}$ is a symmetric matrix with all real entries, and therefore a Hermitian matrix. In this research, $\mathbf{L}$ being a Hermitian matrix is always met with equality since the diagonal entry of each row in $\mathbf{L}$ is the degree of node $i$, and each link connected to $i$ results in -1 in the same row. So the sum of all off diagonals in a row is $d_{i}$. Therefore $\mathbf{L}$ is a positive semi-definite matrix. Since $\mathbf{L}$ is semi-definite (and therefore also Hermitian), the following ordering convention for the eigenvalues will be adopted:

$$
0=\lambda_{1} \leq \lambda_{2} \leq \cdots \lambda_{n}
$$

The interval in which the synchronized state is stable is larger for a smaller ratio of the two eigenvalues $Q=\lambda_{n} / \lambda_{2}$, therefore a network has a more robust synchronized state if the eigenvalue ratio $Q=\lambda_{\mathrm{n}} / \lambda_{2}$ is smaller. Focusing on the first part, the network optimization will be the guided evolution of networks subject to some constraints. Attempting to explain the formation of a small-scale size of networks, the constrained evolution of networks towards consensus or synchrony optimality will be investigated. Constraint optimizations are included via a fitness function that combines the desired goal such as synchronization properties of the networks optimized, or the propensity of a network to synchronize with an average degree requirement needed to connect the nodes.

Ramanujan graph is known in the literatures as the best networks for fast consensus. Ramanujan graphs [16] are $k$ random regular networks with the second minimum eigenvalue satisfying:

$$
\lambda_{2}(L) \geq k-2 \sqrt{k-1},
$$

It is known that among the second minimum eigenvalue $\lambda_{2}(L)$ of various Laplacian matrices, Ramanujan graphs have the largest $\lambda_{2}(L)$ [17]. One class of Ramanujan graphs is a random regular network, which is easy to construct and used in many application for better consensus.

However, for sparse networks with lower average degree, the condition in (7) does not carry any information especially for $k=2$. In this case a Ramanujan graph is a ring network with the degree of 2 as shown in Fig.1(a). However, the second minimum eigenvalue $\lambda_{2}(L)$ of the ring network is very small, and consensus is very slow on the Ramanujan graph with the degree $\mathrm{k}=2$.

Genetic algorithms have been extensively used in single objective optimization for various communication network related optimization problems. Optimizing complex networks usually involve multiple objectives such as the network size as well as various network properties. In this paper, an evolutionary algorithm involving minimization of the eigenvalue ratio of the Laplacian matrix with the constraint of the average degree is used in order to design the optimal network.

Now consider an optimization approach to design networks with better consensus. Although optimization requires finding an optimal solution for a well-defined objective function, optimization as a selection pressure to minimize the following fitness function is considered [18]:

$$
E(\omega)=\omega \frac{\lambda_{n}}{\lambda_{2}}+(1-\omega)\langle k\rangle
$$

where $\langle k\rangle$ is the average degree, and $\omega(0 \leq \omega \leq 1)$ is a parameter controlling two objects.

The eigenvalue ratio $Q=\lambda_{n} / \lambda_{2}$ decreases as the average degree increases and the convergence speed becomes much faster. Therefore an interesting question is how to design a sparse network with a few degrees that guarantees a certain convergence performance. However this is a very difficult combinatorial problem. Therefore, an evolutionary design method is an effective way to design such a sparse optimal network.

Initially 10 random networks with the Poisson degree distributions are generated and the genetic algorithm to obtain better networks in terms of improving the fitness function in (8) is used. The network is encoded as a binary adjacency matrix to perform the mutation and crossover. Next, the most suitable matrices among the parents and children matrices are chosen, and the others are eliminated.

The multi-point crossover was used. After the crossover, each element in the matrix switches to a reverse state with a specific probability. In this paper, the network is an undirected graph, and so, if one element is reversed, the symmetry element is reversed at the same time.

There is a possibility that an isolated network appears after crossover and mutation. In this paper, when an isolated node appears in a new network that the node has infinite distances to another node, the network is dumped. Therefore, non-isolated matrices can be used. After many generations have passed, an optimal network which minimizes the fitness function defined in (8) can be obtained. For dense networks with the average degree $\langle k\rangle$ is larger than 4 , the evolutionary optimized networks are Ramanujan graphs.

However for a sparse network of the average degree $\langle k\rangle=2$, an evolutionary optimized network is a ring-trees type, where many modules networks with tree structures are combined by a ring network. Fig. 1 illustrates the difference of the network topology of an evolutionary optimized network (Fig. 1(b)) from a Ramanujan graph (ring network with $k=2$ ) (Fig. 1(a)).

\section{Simulation Results on CONSENSUS Formations}

In this section, simulation results on fastest and slowest consensus formation on an evolutionary optimized network and a heuristically designed network are shown.

\section{A. Fastest Consensus formation on a sparse network with the average degree $\langle k\rangle=2$}

To examine how fast the process is for achieving consensus among agents in a network, the convergence speed is measured. In the following (9), various initial values are given to each node.

$$
x_{i}(0)=i(i=1,2, \cdots, n)
$$




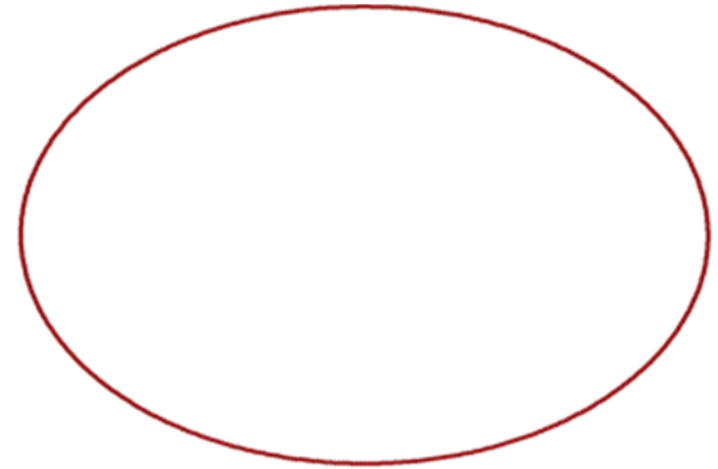

(a)

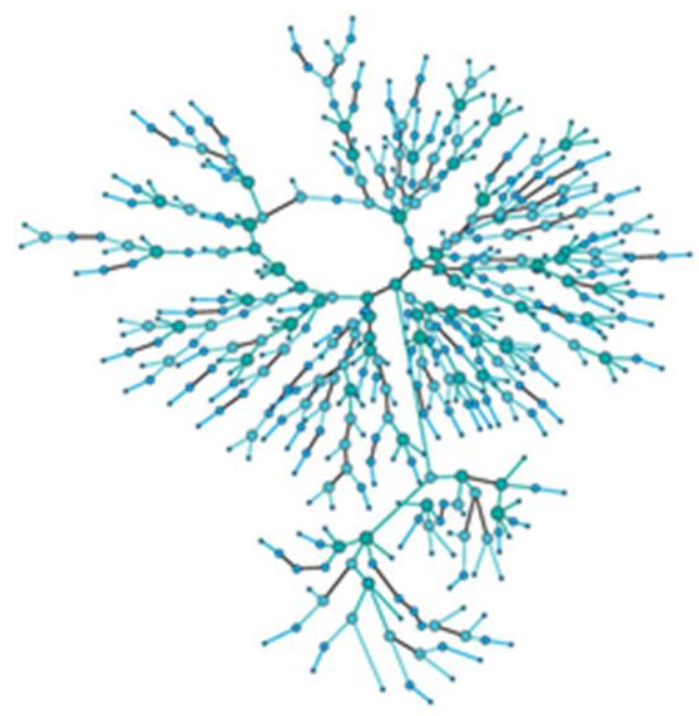

(b)

Fig.1. The convergences of the optimized network for (a) Ramanujan network with $\langle\mathrm{k}\rangle=2$ and (b) ring-trees network.

The state of each agent (node) $x_{i}(1 \leq i<n)$ in the system converges to a constant value. The time required for achieving a consensus is compared, that is, until the states asymptotically converge to the same internal state by way of an agreement characterized by the following equation:

$$
x_{1}=x_{2}=\cdots=x_{n}
$$

Fig. 2 shows the convergences of the optimized network. This means the collective dynamics converge to the average of the initial states of all agents. In general, the optimized network is faster than the Ramanujan network for $\langle k\rangle=2$, the fastest among the previously network models.

Fig. 2 (a) is from Ramanujan network and Fig. 2 (b) is from ring-trees network as shown in Fig. 1 with 100 nodes and 100 links. Consensus dynamics on ring-trees network is achieved much faster than Ramanujan network, where the time of consensus is 1201 steps on ring-trees network and over 5000 steps on Ramanujan network. On the ring-trees network, where agents are divided into each tree network, local consensus is promoted in each tree network at first. After that, the global consensus is formed via the ring network. It is very interesting

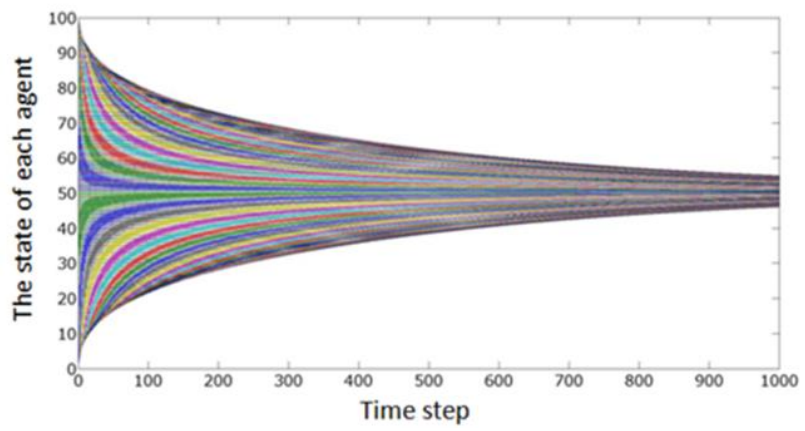

(a)

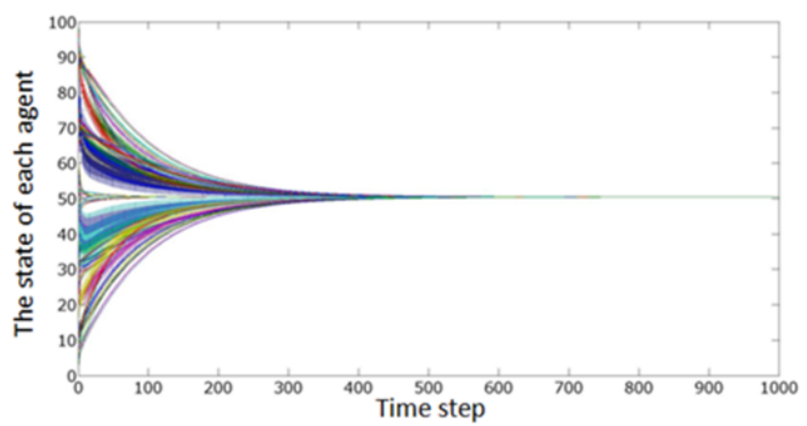

(b)

Fig.2. Network topologies of (a) a Ramanujan graph and (b) an evolutionary optimized network, a ring-trees network, both with 500 nodes and 500 links with an average degree $\langle k\rangle=2$.

that this simple mechanism makes a big difference between these networks on the convergence time of consensus dynamics.

\section{B. Network with the slowest consensus formation}

In this section a heuristic network for slow consensus is proposed. The original topology resulted from the evolutionary design approach. For the slowest design, the inverse of the eigenvalue ratio $\lambda_{2} / \lambda_{n}$ is minimized under the constraint of the average degree:

$$
F(\omega)=\omega \frac{\lambda_{2}}{\lambda_{n}}+(1-\omega)\langle k\rangle
$$

Then the network which minimizes the fitness function in (11) is obtained. Then the optimal network for the slowest consensus is a clique-with-line network as seen in Fig. 3.

The essence of the evolutionary optimized network is extracted and that lead to the following heuristic network design. The line network with a single clique has $N_{M}$ nodes as the dense core and $N_{\text {Line }}$ nodes in the line network. There are $L_{M}$ links in the clique and $L_{\text {Line }}$ links in the line network. If the total resource of building network is limited by $n$ nodes and $L$ links, the following relationships between variants are obtained as,

$$
\begin{aligned}
& n=N_{M}+N_{\text {Line }} \\
& L=L_{M}+L_{\text {Line }}
\end{aligned}
$$




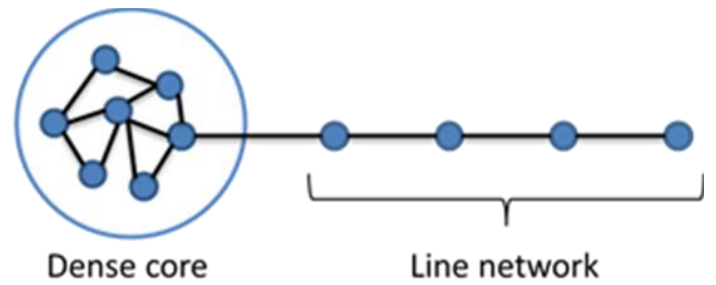

Fig.3. The interconnected network with a clique and a line network

$$
L_{\text {Line }}=N_{\text {Line }}
$$

When the length of line network with $N_{\text {Line }}$ nodes is given in addition to total nodes and total links ( $n$ and $L$ ), the $N_{\text {Line }}$ should meet the following condition to avoid disconnected network and double links between same two nodes.

$$
n-N_{\text {Line }}-1 \leq L-N_{\text {Line }} \leq_{N-N_{\text {Line }}} C_{2}
$$

Now the consensus formation on this optimal network for the slowest consensus formation is evaluated. The internal state value of each agent is plotted over time in Fig. 4(a). Consensus formation is very slow and it takes steps 100 times more than a ring-tree network in Fig. 1(b). The changes of the agent states at the beginning are shown in Fig. 4(b). This figure shows that consensus is achieved among agents in the clique first, then among agents on the line, and globally in the end.

\section{CONSENSUS Formation ON A SYMMETRIC DUMBBELL NETWORK}

Now consider consensus formation in a network with community structures. As a typical example, a "dumbbell (or barbell) graph" with two cliques of identical network structure connected by a single link is used. Since the cliques used here are complete graphs, a Ramanujan [19], any node can be chosen to connect the two cliques. The bridging nodes on both cliques are strategically important since they are the only nodes that connect the two cliques. Additionally, these bridging nodes act as a bottleneck [20] since all nodes in the clique are indirectly influenced by the opposite clique only through their bridging node. Such model may be considered to represent two business teams working together for a common goal.

A graphical image of a dumbbell graph is sown in Fig. 5. Each clique has 50 nodes and 1,225 links therefore 100 nodes and 2,451 links in total. The consensus performance is sown in Fig. 6. The initial values assigned to one clique is $\leq 50$ and to the other clique is $50<$. The progression of consensus in Fig. 6 shows that consensus is formed within the clique before a global consensus is achieved through the bridging nodes and the bridging link. The time it took for consensus is 9,961 steps.

Since the graph used in each clique is a complete graph where all agents are connected to the rest of the agents, the

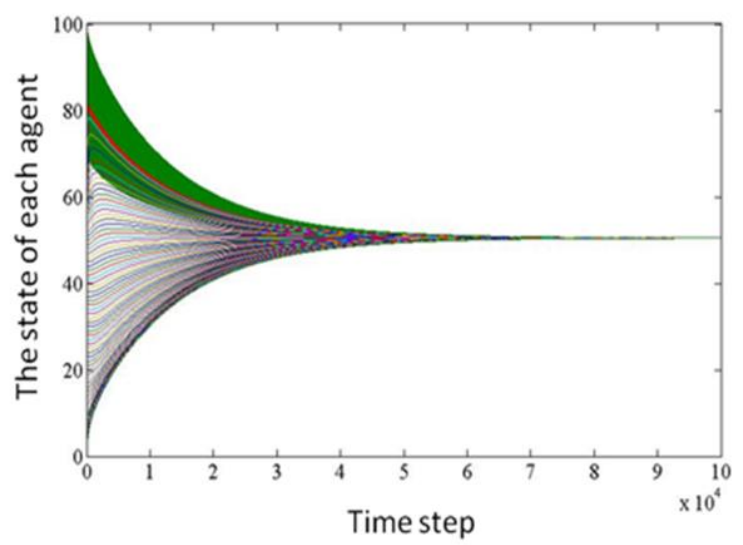

(a)

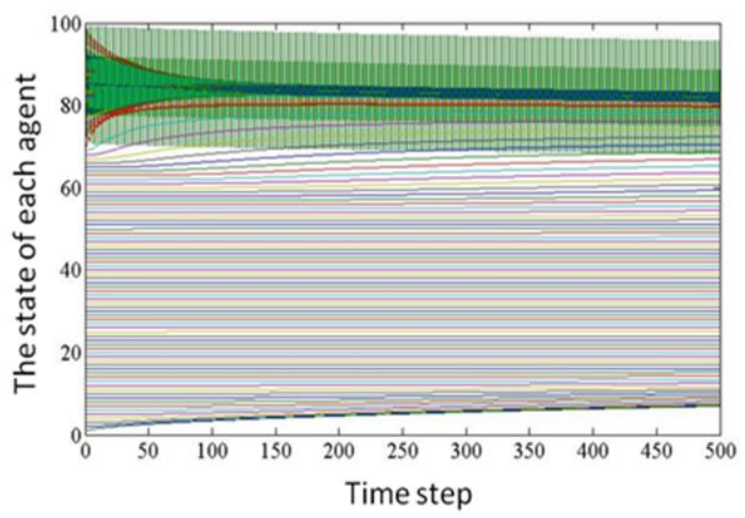

(b)

Fig.4. The diagram of consensus dynamics between agents on a cliquewith-line network with $N=100$ nodes and $L=500$ links. The number of agents in a single core is 34 . In this case the number $i$ is assigned to agent $i$ initially. The plot range of time step is $\left[0,10^{5}\right]$ for (a) and $[0,500]$ for (b).

consensus formation is instant if the cliques were on their own [21]. Although the fastest consensus is achieved in both graphs if they are separated, the consensus becomes very slow if they are connected only by a few links. Therefore the consensus

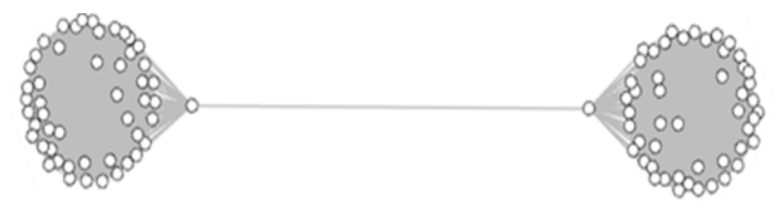

Fig.5. A diagram of a symmetric dumbbell graph. Each clique is composed of 50 agents. The graph contains a total of 100 nodes and 2,451 links. Since each clique is a complete graph, a Ramanujan, the bridging node may be chosen arbitrarily.

dynamics is an example to illustrate that the network dynamics will be affected by the local topological properties as well as the global properties of the network. 


\section{CONCLUSION AND EXTENDED WORKS}

Consensus and synchronization problems are associated with various issues, therefore, the optimal networks proposed prove themselves as one of the best network structures in terms of significance and effectiveness. Designing desirable networks is complex and it may pose a multi-constraint and multicriterion optimization problem.

This paper presented a genetic optimization approach to design an optimal network for consensus formation and synchronization while simultaneously minimizing the eigenvalue ratio and the link density. The convergence speed of evolutionary optimized networks both for fastest convergence and slowest convergence for $\langle k\rangle=2$ was investigated. Additionally, the priority of consensus formation within the network with community structures was observed.

Evolutionary optimized networks for faster consensus is made of a ring and many tree structures that are connected to various nodes on the ring. Although Ramanujan networks are known to have fast consensus characteristics, the optimized network is neither a Ramanujan graph nor random regular networks in which all nodes have the same degrees. Most of the nodes have the same degrees but some nodes have more links and other nodes have less links than the majority of nodes. For slow consensus, a core-with-line network was obtained where one end of a string of nodes is a dense clique with many nodes. Finally, two clique graphs were connected by a single link and its consensus property was observed. The consensus dynamics of the above networks show that consensus is formed among agents with denser links first, then a global consensus is formed. This demonstrates that both local and global topological properties contribute to the overall behavior of the network dynamics.

As for future development of this research based on the above results obtained, solving a network design problem of additional complexity by including larger number of objectives and constraints is an area of further investigation. The constraints may differ for networks in a geographic setting in addition to a topological layout, as in the case with real networks such as the internet and the brain cell network. For additional constraint, not only the wiring cost but also the maintenance of links in real distances may be used [22].

Many networks occurring in real life have modular structures that are arranged in a hierarchical structure. A more complex consensus model can also be considered where each module can represent a community of various interests [23] as the dumbbell network, or even a module as a single agent with distinct parameters represented by each node, with thresholds for each node in order to gain consensus within the module of nodes. Such a setting may be used to model an individual's psychology and their motivational level especially when there are many factors to be weighed in a decision making process.

Still further ahead, how to design ultra-large networks with the optimal principle will be investigated. Optimal networks (network modules) are achieved by treating each module as a node that has the same degree distribution as the rest of the optimal modules. The connecting nodes of two modules are selected stochastically in proportion to the node positions in

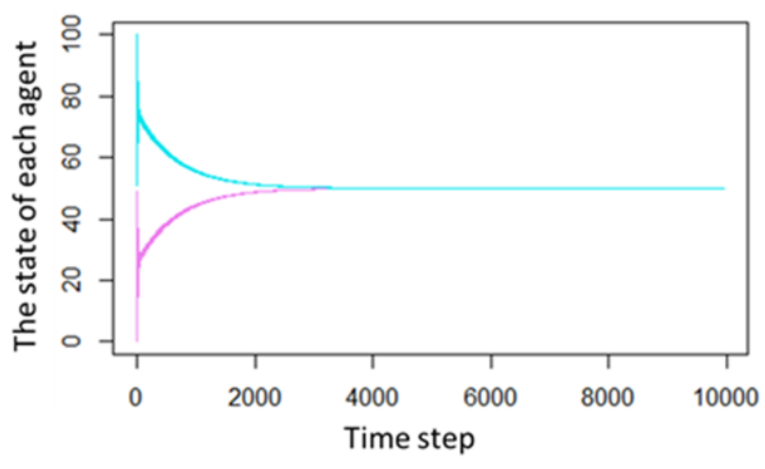

Fig.6. A consensus progression diagram for the dumbbell graph in Fig. 5. The initial values assigned to agents in each clique are first half of 100 to one clique and the second half of 100 to the other clique.

each module. The benefit of such modularization of a network is that the sparse connectivity between modules can prevent the contagion of risk spreading [24]. Observation of consensus dynamics and speed can reflect the robustness of large modularized optimal networks.

\section{REFERENCES}

[1] J. Surowiecki, The Wisdom of Crowds, New York City, NY: Random House, 2004.

[2] J. Lorenza, H. Rauhutb, F. Schweitzera, and D. Helbingb, "How social influence can undermine the wisdom of crowd effect," PNAS, vol. 108, no. 22, pp.106-112, 2011.

[3] A. Pikovsky, M. Rosenblum, and J. Kurths, Synchronization: A Universal Concept in Nonlinear Sciences, Cambridge, Cambridge University presses, 2003.

[4] R. Olfati-Saber, J. A. Fax, and R. M. Murray, "Consensus and cooperation in networked multi-agent systems", Proceedings of the IEEE, vol. 95, no. 1, pp.215-233, 2007.

[5] A. Adamatzky, Dynamics of Crowd-Minds, Singapore, World Scientific, 2005.

[6] D. J. Watts and S. H. Strogatz, "Collective dynamics of small-world networks," Nature, vol. 393, pp. 440-442, 1998.

[7] A. L. Barabási, Z. Dezso, E. Ravasz, S. H. Yook, and S. Oltvai, "Scalefree and hierarchical structures in complex networks," Modeling of Complex Systems: Seventh Granada Lectures, vol. 661, pp.1-16, 2003.

[8] S. H. Strogatz, "Exploring complex networks," Nature, vol. 410, no. 6825, pp.268-276, March 2001.

[9] A. L. Barabási, Linked: The New Science of Networks, New York city, NY: Perseus Publishing, 2002.

[10] L. Donetti, P. I. Hurtado, and M.A. Munoz, "Entangled networks, synchronization and optimal network topology," Physical Review Letters, vol. 95, 188701, 2005.

[11] I. Belykh, M. Hasler, M. Lauret, and H. Nijmeijer, "Synchoronization and graph topology," International Journal of Bifurcation and Chaos, vol. 15, no. 11, pp. 3423-3433, 2005.

[12] H. Sato, O. Isao, and S. Kobayashi, "A new generation alternation model of genetic algorithms and its assessment," Journal of Japanese Society for Artificial Intelligence, vol. 12, no. 5, pp. 734-744, 1997.

[13] B, Golub and M. Jackson, "Naïve learning in social networks and the wisdom of crowds," American Economic Journal: Microeconomics, vol. 2, pp.112-149, 2010.

[14] M. H. DeGroot, "Reaching a consensus," Journal of the American Statistical Association, vol. 69, iss. 345, pp. 118-121, 1974.

[15] L. Xiao and S. Boyd, "Fast linear iterations for distributed averaging," Systems \& Control Letters, vol. 53, no. 1, pp. 65-78, 2004.

[16] A. Lubotzky, R. Phillips, and P. Sarnak, "Ramanujan graphs," Combinatorica, vol. 8, no. 3, pp. 261-277, 1988. 
[17] S. Kar and J. M. F. Moura, "Topology for global average consensus," Signals, Systems and Computers, pp. 276-280, 2006.

[18] T. Komatsu and A. Namatame, "Distributed Consensus and Mitigating Risk Propagation in Evolutionary Optimized Networks," J.-H. Kim, et al. (Eds.): AsiaSim2011, PICT 4, Springer, pp. 200-209, 2012.

[19] M. R. Murty, "Ramanujan graphs", J. Ramanujan Math. Soc., vol. 18, pp. 1-20, 2003.

[20] S. Sreenivasan, R. Cohen, E. Lopez, Z. Toroczkai, and H. E. Stanley, "Structural bottlenecks for communication in networks," Physical Review E, vol. 75, 036105, 2005.
[21] F. Fagnani, "Consensus dynamics over networks," 2014.

[22] R. K. Pan and S. Sinha, "Modular networks with hierarchical organization: The dynamical implications of complex structure," Pramana Journal of Physics, vol. 71, no. 2, pp. 331-340, 2008.

[23] J. Gao, S. Buldyrev, E. Stanley, and S. Havlin, "Networks formed from interdependent networks," Nature Physics, vol. 8, pp. 40-48, 2012.

[24] T. Komatsu and A. Namatame, "An evolutionary optimal network design to mitigate risk contagion," in Proc. The 7th International Conference on Natural Computation,, IEEE, pp. 1980-1985, 2011. 